\title{
Isolated Glossoplegia in Capsular Infarction
}

\author{
Ji Hoon Lee, Dokyung Lee, Sung Hyuk Heo, Dae-il Chang
}

Keywords: cerebrovascular disease, neuroanatomy, stroke, stroke imaging

doi:10.1017/cjn.2015.353

Can J Neurol Sci. 2016; 43: 843-844

A 68-year-old healthy woman presented with sudden onset of slurred speech and difficulties in mastication. On admission, when asked to protrude her tongue, the tongue was deviated to right side. (Figure 1) However, the tongue showed normal range of motion at rest and while moving in other directions. Neurological examination was normal, including other cranial nerves and motor and sensory functions of extremities. She denied other symptoms such as headache, nausea, fever or visual disturbance. In addition the initial serologic test showed normal result, including inflammatory markers. Diffusion weighted images demonstrated a focal acute ischemic lesion in left posterior limb of the internal capsule (PLIC). (Figure 2) There were no other abnormal findings on fluid attenuation inversion recovery images and magnetic resonance angiogram. Her symptoms improved gradually and she was discharged at seven days after admission without any neurological sequelae.

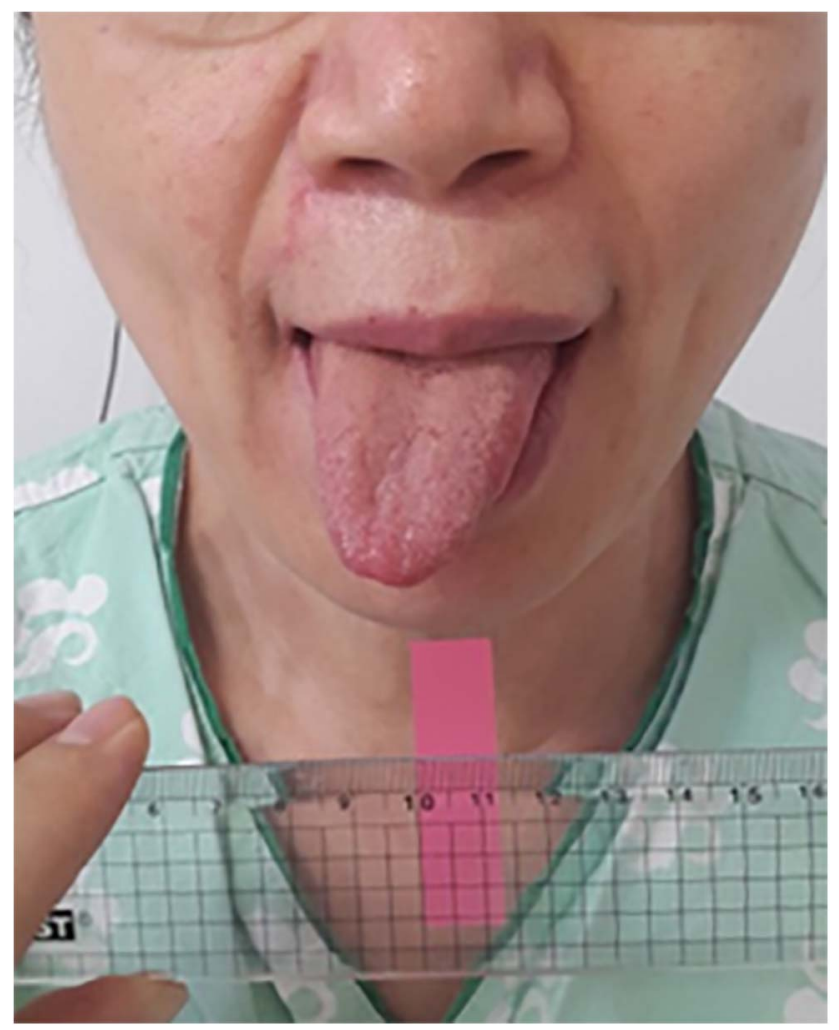

Figure 1: Right lingual paresis evident on tongue protrusion (at admission). At rest and while moving in other directions, the tongue showed normal range of motion. (not shown).

\section{Discussion}

Lingual hemiparesis can result from damage to both upper and lower motor neurons. However, the clinical presentation from each location may be different. Because each hypoglossal nucleus is innervated from both sides of the motor cortex simultaneously, unilateral lesion of the upper motor neuron seldom presents a contralateral lingual paresis. In addition, isolated lingual hemiparesis needs to be differentiated from other diagnosis, such as infarction or neoplasm in the medulla, structural lesion at cistern or hypoglossal canal or acute carotid artery dissection.

Among the extrinsic muscles of the tongue, only the genioglossus (the tongue protrudor muscle) has a crossed unilateral innervation. ${ }^{1}$ For this reason, the lingual hemiparesis resulting from a contralateral lesion in the upper motor neurons can be more evident when the tongue is protruded, presenting as contralesional deviation. To our knowledge, one case report about an isolated lingual hemiparesis from a hematoma at contralateral PLIC has been previously published. ${ }^{2}$

The somatotopic organization of motor fibers within the PLIC has been extensively studied but still remains unclear. According to a previous description about capsular genu syndrome, the motor corticopotine and corticobulbar fibers tend to be situated closer to the genu of the internal capsule. ${ }^{3}$ This is further supported by more recent studies, which show that fibers from tongue motor cortices are situated more anteriorly than fibers from the arm and leg motor cortices within the PLIC. ${ }^{4,5}$ In our case, however, the isolated lingual hemiparesis resulted from a lesion which was situated more posteriorly and distant from the genu, than previously expected. This difference may come from the anatomical diversity in which the courses and overlap between motor fibers may differ from person to person.

Therefore, we suggest that isolated lingual hemiparesis could result from a focal lesion in PLIC even if the lesion is not directly adjacent to the genu.

In conclusion, this case demonstrates the precise somatotopic location of motor fibers of the tongue by a focal ischemic lesion on magnetic resonance imaging.

\section{DisClosures}

Ji Hoon Lee, Dokyung Lee, Sung Hyuk Heo, and Dae-il Chang do not have anything to disclose.

From the Department of Neurology, Kyung Hee University College of Medicine, Seoul, Korea.

Correspondence to: Dae-il Chang, Department of Neurology, Kyung Hee University Hospital, 23 Kyungheedae-ro, Dongdaemun-gu, Seoul 130-872 Republic of Korea. Email: dichang@khmc.or.kr 


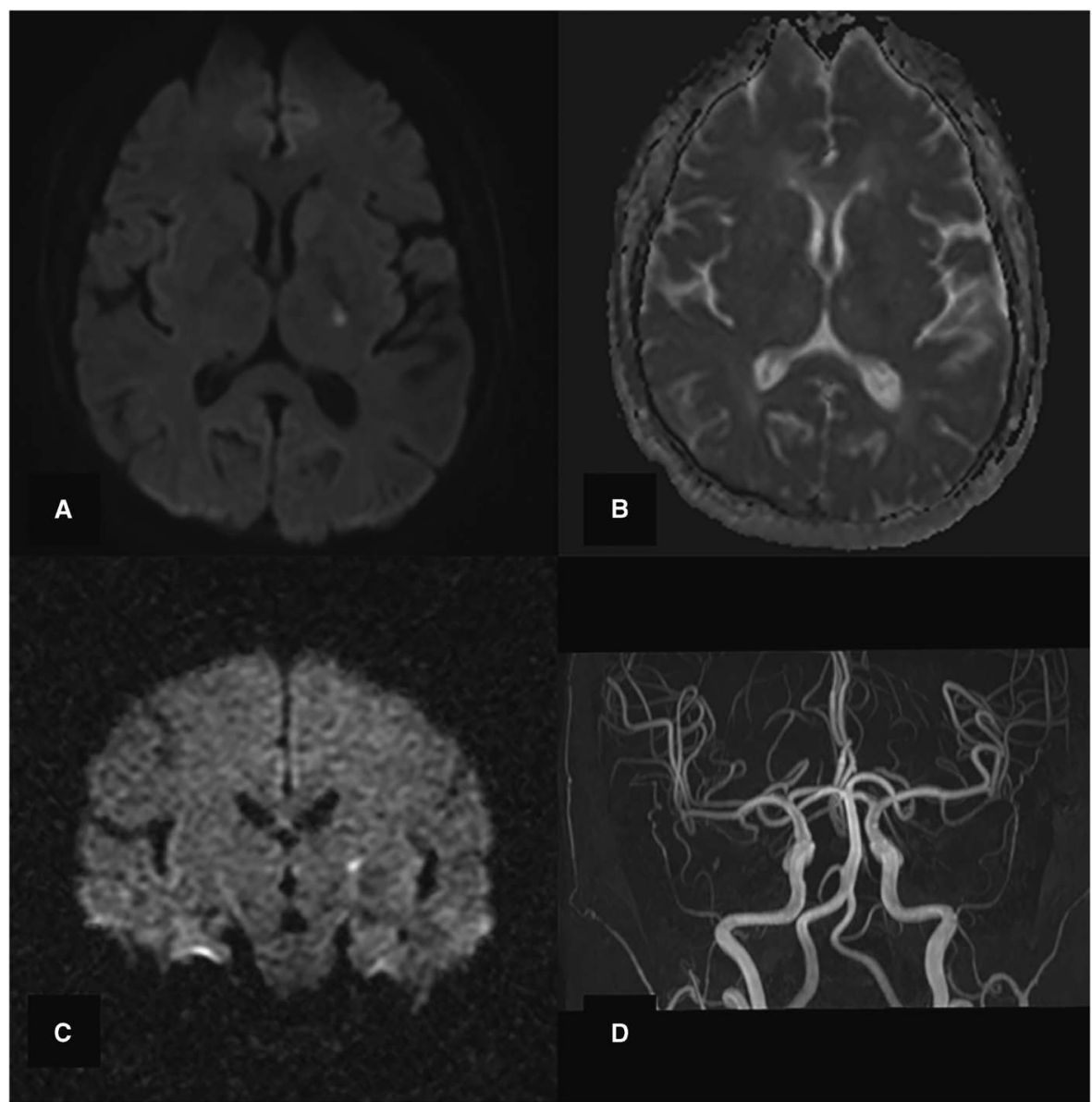

Figure 2: Focal acute ischemic lesion in posterior limb of left internal capsule, on MRI A) axial diffusion weighted images (DWI). B) apparent diffusion coefficient $(A D C)$, C) coronal DWI. $D$ ) There were no other abnormal findings on FLAIR images (not shown) and MRA.

\section{REFERENCES}

1. Mu L, Sanders I. Human Tongue Neuroanatomy: Nerve Supply and Motor Endplates. Clin Anat. 2010;23:777-91.

2. Gerace C, Fele RM, Pingi A. Capsular lingual paresis. J Neurol Neurosurg Psychiatry. 2005;76:595.

3. Bogousslavsky J, Regli F. Capsular genu syndrome. Neurology. 1990;40:1499-502
4. Pan C, Peck KK, Young RJ, et al. Somatotopic organization of motor pathways in the internal capsule: a probabilistic diffusion tractography study. Am J Neuroradiol. 2012;33:1274-80.

5. Duerden EG, Finnis KW, Peters TM, et al. Three-dimensional somatotopic organization and probabilistic mapping of motor responses from the human internal capsule. J Neurosurg. 2011;114:1706-14. 